\title{
Computational inference of H3K4me3 and H3K27ac domain length
}

Julian Zubek, Michael L Stitzel, Duygu Ucar, Dariusz M Plewczynski

Background. Recent epigenomic studies have shown that the length of a DNA region covered by an epigenetic mark is not just a byproduct of the assaying technologies and has functional implications for that locus. For example, expanded regions of DNA sequences that are marked by enhancer-specific histone modifications, such as acetylation of histone H3 lysine 27 (H3K27ac) domains coincide with cell-specific enhancers, known as super or stretch enhancers. Similarly, promoters of genes critical for cell-specific functions are marked by expanded H3K4me3 domains in the cognate cell type, and these can span DNA regions from $4-5 \mathrm{~kb}$ up to $40-50 \mathrm{~kb}$ in length. These expanded H3K4me3 domains are known as buffer domains or super promoters.

Methods. To ask what correlates with-and potentially regulates-the length of loci marked with these two important histone marks, H3K4me3 and H3K27ac, we built Random Forest regression models. With these models we computationally identified genomic and epigenomic patterns that are predictive for the length of these marks in seven ENCODE cell lines.

Results. We found that certain epigenetic marks and transcription factors explain the variability of the length of $\mathrm{H} 3 \mathrm{~K} 4 \mathrm{me} 3$ and H3K27ac marks across different cell types, which implies that the lengths of these two epigenetic marks are tightly regulated in a given cell type. Our source code for the regression models and data can be found at our GitHub page: https://github.com/zubekj/broad_peaks

Discussion. Our Random Forest based regression models enabled us to estimate the individual contribution of different epigenetic marks and protein binding patterns to the length of $\mathrm{H} 3 \mathrm{~K} 4 \mathrm{me} 3$ and $\mathrm{H} 3 \mathrm{~K} 27 \mathrm{ac}$ deposition patterns; therefore potentially revealing genomic signatures at cell specific regulatory elements. 
1 Computational inference of H3K4me3 and H3K27ac

2

3

4

5

$6{ }^{1}$ Centre of New Technologies, University of Warsaw, Poland

$7 \quad 2$ Institute of Computer Science, Polish Academy of Sciences, Poland

$8{ }^{3}$ The Jackson Laboratory for Genomic Medicine, Farmington, Connecticut, Unites States of 9 America

10

11

12

13

14

15

16

17

18

19

20

21

22

23

\section{domain length}

\author{
Julian Zubek ${ }^{1,2}$, Michael Stitzel ${ }^{3}$, Duygu Ucar ${ }^{3 *}$, Dariusz Plewczynski ${ }^{1^{*}}$
}

\section{Corresponding authors*:}

Dariusz Plewczynski

Centre of New Technologies, University of Warsaw, Poland

e-mail: d.plewczynski@cent.uw.edu.pl

Duygu Ucar,

The Jackson Laboratory for Genomic Medicine, Farmington, Connecticut, Unites States of America

e-mail: Duygu.ucar@jax.org 
25

26

27

28

29

30

31

32

33

34

35

36

37

38

39

40

41

42

43

44

45

46

47

48

49

50

51

52

53

54

55

56

57

58

59

60

61

62

63

64

65

\section{Abstract}

Background. Recent epigenomic studies have shown that the length of a DNA region covered by an epigenetic mark is not just a byproduct of the assaying technologies and has functional implications for that locus. For example, expanded regions of DNA sequences that are marked by enhancer-specific histone modifications, such as acetylation of histone H3 lysine 27 (H3K27ac) domains coincide with cell-specific enhancers, known as super or stretch enhancers. Similarly, promoters of genes critical for cell-specific functions are marked by expanded $\mathrm{H} 3 \mathrm{~K} 4 \mathrm{me} 3$ domains in the cognate cell type, and these can span DNA regions from 4-5kb up to 40-50kb in length. These expanded H3K4me3 domains are known as buffer domains or super promoters.

Methods. To ask what correlates with-and potentially regulates-the length of loci marked with these two important histone marks, H3K4me3 and H3K27ac, we built Random Forest regression models. With these models we computationally identified genomic and epigenomic patterns that are predictive for the length of these marks in seven ENCODE cell lines.

Results. We found that certain epigenetic marks and transcription factors explain the variability of the length of H3K4me3 and H3K27ac marks across different cell types, which implies that the lengths of these two epigenetic marks are tightly regulated in a given cell type. Our source code for the regression models and data can be found at our GitHub page: https://github.com/zubekj/broad_peaks

Discussion. Our Random Forest based regression models enabled us to estimate the individual contribution of different epigenetic marks and protein binding patterns to the length of H3K4me3 and H3K27ac deposition patterns; therefore potentially revealing genomic signatures at cell specific regulatory elements.

\section{Introduction}

Epigenomics refer to the heritable changes that are not stemming from the changes in the genomic DNA sequence. The major epigenetic mechanisms can be categorized into two distinct classes: 1) methylation of the cytosine residues of DNA (i.e., DNA methylation); and 2) the posttranslational modifications of the histone proteins (i.e., histone modifications). Recent advancements in next generation sequencing (NGS) technologies have enabled the development of experimental methods to generate genome-wide profiles of epigenetic marks in many types of cells and organisms. These technologies enable profiling epigenetic states genomewide as collections of short sequence reads associated with a given epigenetic mark, which is then aligned to the reference genome. Each locus in the genome has therefore an associated read count, where regions with high abundance of reads (i.e., peaks) are captured to identify loci marked by the profiled epigenetic mark. It has been previously shown that the length of a particular locus covered by an epigenetic mark is related to the cell-specific functions of that region. For example, several research groups observed that long genomic segments marked by enhancer-specific histone modifications, such as acetylation of histone $\mathrm{H} 3$ lysine 27 (H3K27ac), are found at cell-specific enhancer loci [1, 2, 3]. Similar observations have been made for DNA methylation profiles. Continuous regions of low DNA methylation (at least 
66 five kilobase ( $\mathrm{kb}$ ) in length), namely DNA methylation valleys or canyons, have been associated 67 with transcription factors and with genes known to regulate development in embryonic stem 68 cells and genes with potential involvement in the regulation of hematopoiesis in hematopoietic 69 stem cells (HSCs) [4]. In addition, in a recent publication, we showed that longer domains (from 705 to $50 \mathrm{~kb}$ ) of histone $\mathrm{H} 3$ lysine 4 trimethylation (H3K4me3) preferentially mark genes 71 associated with cell identity and function in diverse cell types and organisms [5]. These 72 expanded H3K4me3 domains, termed broad domains, have also been shown to mark tumor suppressors in normal cells when compared to tumor pairs [6].

These recent studies show that the long continuous segments of DNA covered by H3K4me3 and $\mathrm{H} 3 \mathrm{~K} 27 \mathrm{ac}$ can be used as an epigenetic signature for identifying cell-type-specific promoters and enhancers and these epigenetic patterns might be established and maintained by the cell to robustly regulate cell-type specific gene expression patterns and functions. However, what potentially regulates the deposition or maintenance of such cell-specific epigenetic signatures still remains an open question. Computationally identifying genomic and epigenomic characteristics associated with the length of these histone marks might provide some hints about potential mechanisms. For this purpose, to systematically identify genomic and epigenomic characteristics associated with cell-specific epigenetic signatures, we build Random Forest regression models to explain the length of H3K4me3 and H3K27ac domains using both genomic and epigenomic information. Our regression models are different than our previous method that is mainly comparing very long H3K4me3 domains to shorter domains [5]. Here, we study the length of a domain as a spectrum without arbitrarily introducing a length cut-off. Moreover, we also study H3K27ac patterns in addition to H3K4me3, which is the mark that has been used for identifying cell-type-specific enhancers. To the best of our knowledge, this is the first attempt to study the length of an epigenetic mark under the light of other genomic and epigenomic characteristics of that site. A recent algorithm showed that in the existence of many epigenetic marks, missing epigenetic marks could be imputed at 25-bp resolution using regression tress [7]. Although this method and study was informative in showing that epigenetic marks are not independently deposited from each other, it did not reveal whether any of these correlations are important for the length of H3K4me3 and H3K27ac domains. Moreover, this method was restricted to histone modification profiles and didn't study the relationship between protein binding patterns and the length of these two important epigenetic marks.

99

100

101

Using our regression models in seven ENCODE cell lines (human embryonic stem cell lines $\mathrm{H} 1$ and $\mathrm{H} 9$ and cancer cell lines K562, HCT116, GM12878, A549 and HeLa), we asked whether the

102

103 length of loci marked with H3K4me3 or H3K27ac domains can be predicted using other genomic features of that loci including transcription factor (TFs) binding patterns and other epigenetic marks using chromatin immunoprecipitation followed by sequencing (ChIP-seq) datasets. This enabled us to systematically study the relationship between H3K4me3 and H3K27ac length found at a locus and other genomic features of that locus. Moreover we also quantified the importance of different genomic features in describing the epigenetic domain length. Our analyses revealed that with integrative computational models we could predict the length of H3K4me3 and H3K27ac domains with high efficacy (>0.9 correlation on the average between 
110 observed and predicted). Moreover, with our models we can infer the most important genomic 111 and epigenomic features that are predictive for the length of these two epigenetic marks.

112

\section{Materials \& Methods}

114

ChIP-seq data pre-processing: Publicly available datasets were obtained from ENCODE consortium [8], in multiple ENCODE cell lines as well as in pancreatic islets (accession numbers are listed in Table S1). To preprocess ChIP-seq datasets, we mapped reads to reference human genome (hg19) using bowtie0.12.7 [9]. ChIP-seq peaks were called using MACS2.08 [4] with the "-broad" option for histone marks. Peaks were assigned to the gene with the closest transcription start site (TSS).

\section{Domain length prediction using Random Forest regression algorithm}

122 Feature extraction: For each histone modification domain and data feature being studied, 123 we used the fraction of overlapping basepairs (bps) in our models to eliminate the bias that could be introduced by differences in domain lengths. For example, if an H3K4me3 domain has length 100 base pairs and 15 of these bases also have CHD1 binding, fraction of overlap for that domain for the CHD1 feature is $(15 / 100=0.15)$. Note that a single H3k4me3 domain can simultaneously overlap with multiple instances of the same feature (e.g., multiple CHD1 binding sites within a domain). For these instances, we used the sum of all of such overlaps. For example, as depicted in the Fig. 1C domain B overlaps with two instances of domains A,

Model building: Random Forest regression with 100 trees was performed on the constructed data sets. We employed these models to unfold the relationship between domain length and the constructed features. We used an implementation available in scikitlearn library [10]. Gini impurity was used as a quality measure for choosing best splits. At each level $\sqrt{\mathrm{m}}$ randomly chosen attributes were considered as candidates for split (where $\mathrm{m}$ is the number of all attributes).

Feature ranking: Feature rankings enable identifying and ranking important features for the regression problem at hand. When building a decision tree, the feature that leads to the greatest decrease in Gini impurity score is chosen at each split. Importance score for each feature is the mean decrease of impurity for all tree nodes, which is averaged over all trees in the ensemble. Calculating importance scores enable comparison of attributes, but does not state the significance of importance scores. To assess significance we employed a Monte-Carlo technique based on contrast attributes, which are random permutations of the original attributes. To quantify the significance of a feature importance score, we followed the below procedure:

150

1. For each of the original attributes its values were permuted at random and added as a new contrast attribute to the original dataset.

2. Random Forest model was trained on the dataset consisting of both original and contrast attributes. 
154

155

156

157

158

159

160

161

162

163

164

165

166

167

168

169

170

171

172

3. Standard deviation (SD) of importance scores for contrast attributes was calculated. The value equal to $2 \mathrm{xSD}$ was used as a cutoff for minimal significant difference between importance scores.

4. Feature importance scores were sorted in decreasing order. Differences between subsequent scores were calculated. We looked for the last pair of subsequent scores for which the difference was larger than the cutoff value. The larger of the scores from that pair constituted a threshold for dividing features into significant and not significant.

This procedure enabled us to identify a small set of significant features for our models.

A Distribution of H3K4me3 ChIP-seq peaks

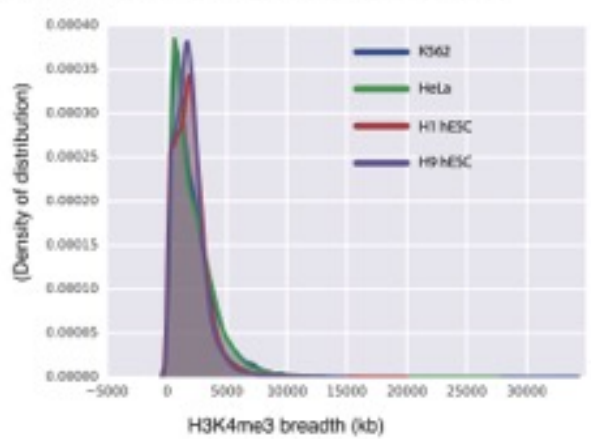

H3K4meg breadth (ab)
B Computational framework

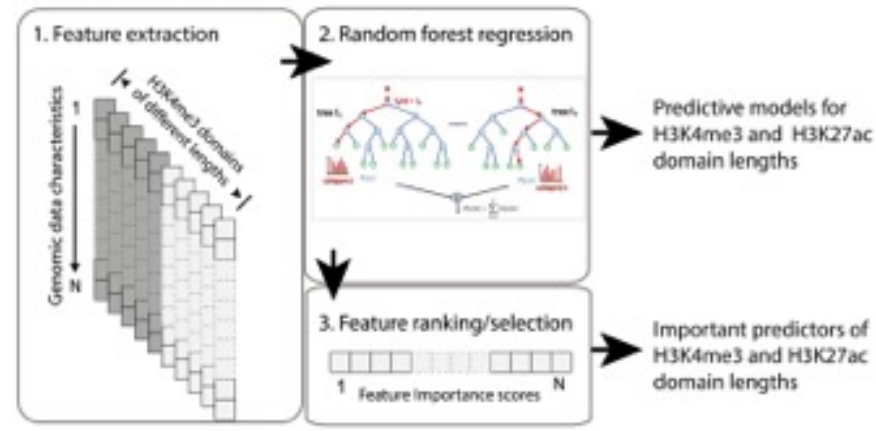

domain lengths

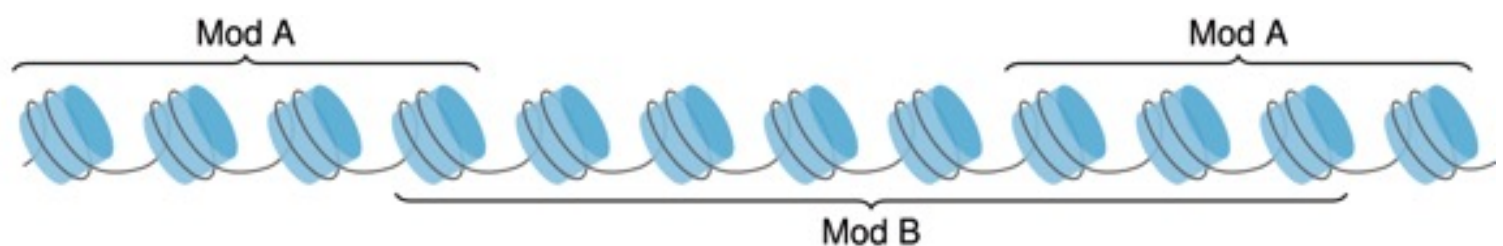

Figure 1: H3K4me3 domain lengths vary. A. H3K4me3 domain length distribution in four ENCODE cell lines. H3K4me3 domain lengths span a wide range and longer domains mark cell-type-specific promoters. B. Our three-step framework to model the length of H3K4me3 and H3K27ac domains. C. An example of domain type B, which overlaps with two instances of domains of type A. 


\section{Results}

174

175

176

177

H3K4me3- and H3K27ac-domain lengths are informative for cell-type specificity

It has been observed that epigenetic marks can decorate loci with domains of varying length.

178 For example, when we study the domain lengths for H3K4me3, we observe that the length

179 distribution is not uniform and the length of these peaks vary between a few hundred and $20 \mathrm{~kb}$ as shown in the length distribution of H3K4me3 domains in $\mathrm{H} 1$ human embryonic stem cells

181

182 (hESCs) (Fig. 1A). The span of epigenetic mark deposition has gained attention in recent years with several studies, including ours, showing that particularly long stretches of DNA marked

184

185

186 with H3K4me3 or H3K27ac specifically coincide with cell-type specific promoters or enhancers, respectively. $[1,2,3,5,11]$.

187

Given the association of large domains with functionally important DNA elements, we built a computational framework that can i) assess whether-and to what extent-the length of an H3K4me3 (or H3K27ac) domain at a locus can be predicted from other genomic and epigenomic characteristics of that locus and ii) quantify the ability of each genomic and epigenomic characteristic to predict the length of domains of these two marks. Our framework consisted of three stages (summarized in Fig. 1B): 1) extracting genomic and epigenomic characteristics of each domain; 2) building a regression model (based on Random Forest regression) for the length of the domains as a function of other data characteristics; and 3) delineating the predictive genomic signature for domain length by prioritizing and selecting important predictors. The signatures we obtained will help us to identify candidate molecular mechanisms for setting or maintaining domain length of this epigenetic mark.

\section{H3K4me3 and H3K27ac deposition lengths can be predicted with high accuracy} by integrating other genomic datasets

200

201

For each H3K4me3 or $\mathrm{H} 3 \mathrm{~K} 27 \mathrm{ac}$ domain, we extracted other genomic and epigenomic characteristics from ENCODE datasets. The complete set of datasets used in each cell type is listed in Fig. 2. We used the fraction of overlap (as explained in methods) to normalize feature overlaps based on domain length, so longer domains would not have an inflation of overlapping features. Next, in seven ENCODE cell lines (H1 and H9 hESCs, K562, HCT116, GM12878, A549, HeLa) as well as in human pancreatic islets, we built Random Forest Regression models to predict the length of H3K4me3 and H3K27ac domains. Our analyses showed that these models can predict the length of epigenetic marks with high accuracy ( $>0.9$ correlation between observed and predicted lengths; Fig. 2).

209
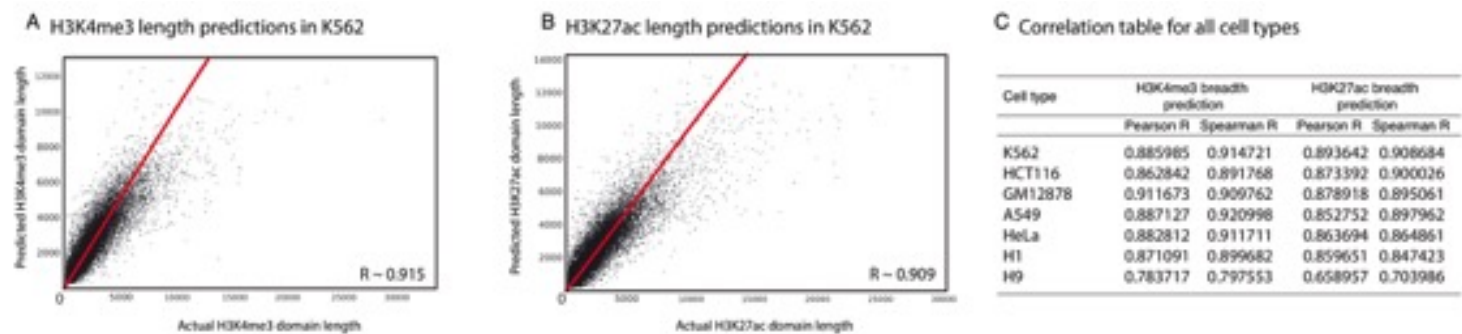

Figure 2: Our computational models can predict the length of H3K4me3 and H3K27ac domains with high precision. A Predicted ve observod H3K1me3 domains using our model in K562. B. Predicted vs. observed H3K27ac

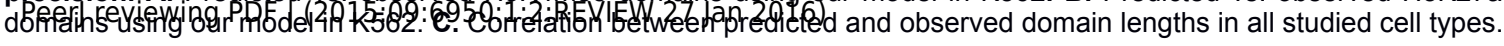


Conserved genomic features are predictive for H3K4me3 and H3K27ac

214 Random Forest quantifies the importance of each feature by calculating its contribution to the 215 overall classification accuracy (see methods for details). We calculated feature importance 216 scores for each genomic and epigenomic data type we used in our models. Next, we assessed 217 the significance of these scores by comparing them with scores produced by random 218 permutations of the original features (i.e., contrast features). With this methodology, we identified the significant predictors of length for H3K4me3 and H3K27ac in each studied cell type. For example, we showed that in $\mathrm{K} 562 \mathrm{CHD} 1$ is one of the most important predictors for both H3K4me3 and H3K27ac domains (Figures 3 and 4).

Our random forest regression models revealed that there are frequently repeating features that are predictive for both H3K4me3 and H3K27ac epigenetic marks, which point out to putative upstream or downstream associations between the length of epigenetic marks and these predictive features. Certain genomic and epigenomic datasets frequently rank as important for predicting the length of these two marks across cell types (Fig. 3C-D). For H3K4me3, CHD1- and Pol2-binding and the H3K79me2 histone modification are the most important predictors as assessed by feature importance scores. For H3K27ac, TEAD1- and CHD1-binding and the presence of other acetylation marks (H3K4ac and H2AK5ac) are important genomic characteristics. 
A H3K4me3 length predictors in K562

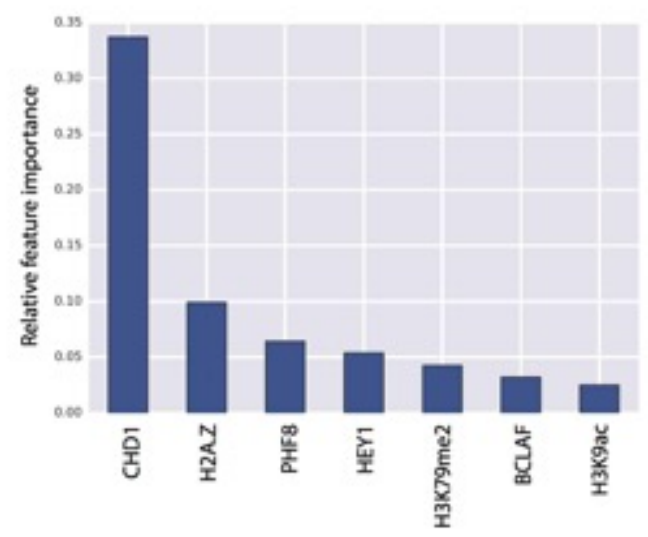

C Feature importance scores (H3K4me3)

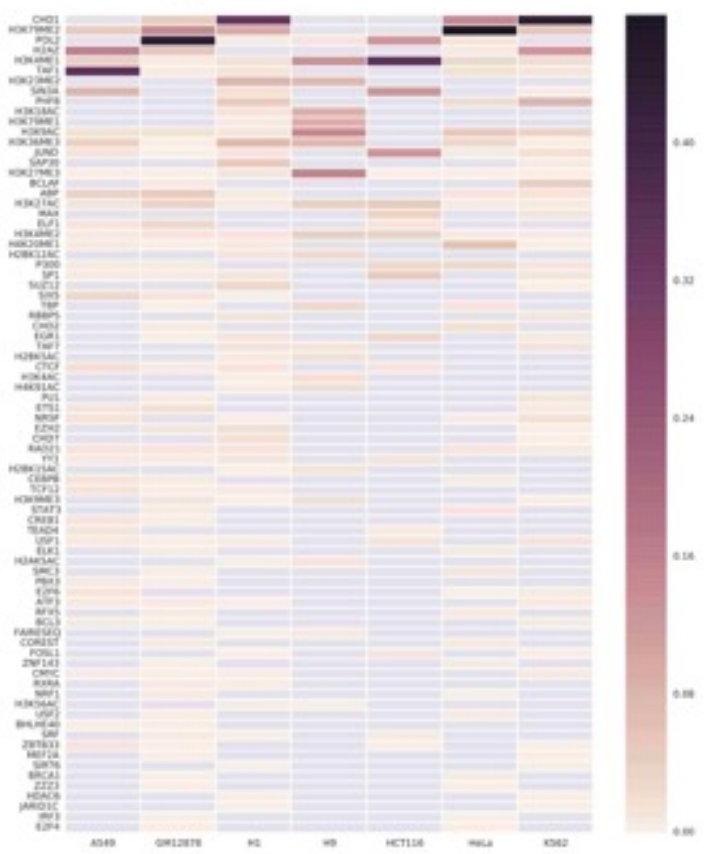

B H3K27ac length predictors in K562

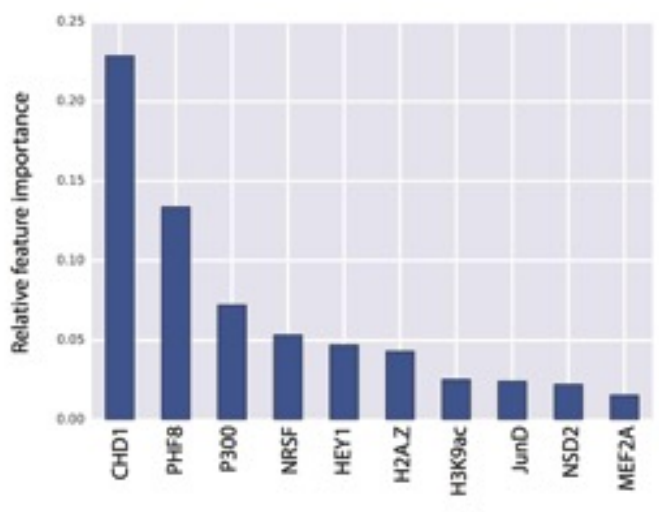

D Feature importance scores (H3K27ac)

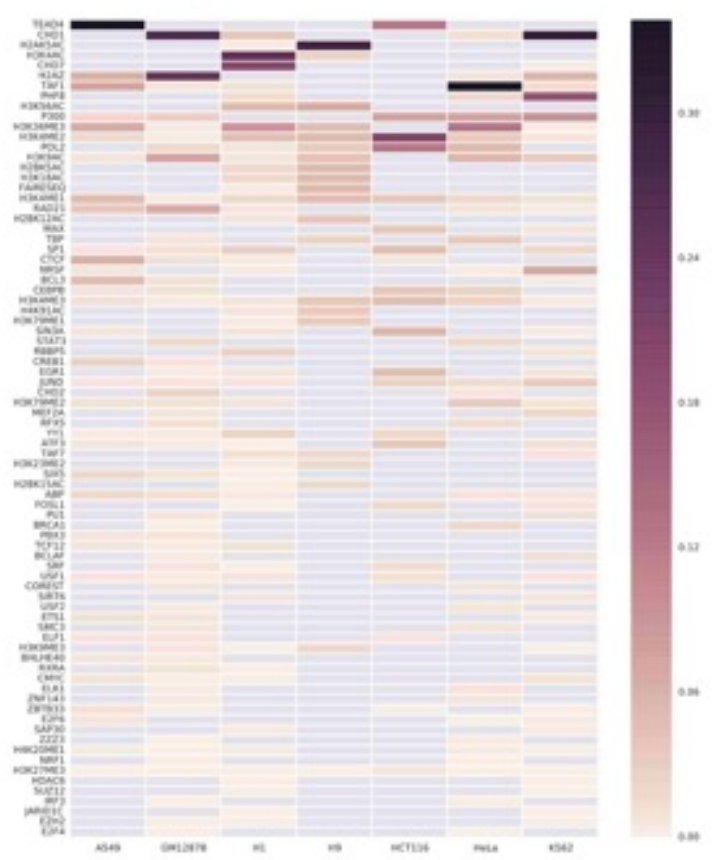

Figure 3: We identified genomic features that play an important role in H3K4me3 and H3K27ac domain lengths. Significant predictors of H3K4me3 domain length in K562 (A) and in all studied cell types (C). Significant predictors of H3K27ac domain length in K562 (B) and in all studied cell types (D). Missing datasets in the studied cell types are represented in white.

Elongated CHD1 binding might promote robust and increased expression patterns

Among the top predictors, CHD1 is an H3K4me3-associated chromatin remodeler and it is shown to be essential for the expression of key developmental genes in mouse embryonic stem (ES) cells as well as for reprogramming of fibroblasts to the pluripotent stem cells [12].

244 Moreover, CHD1 binding is shown to be essential for the assembly of preinitiation complexes 
245 (PIC), which is necessary to start transcription. PIC includes co-activators like Mediator complex 246 and the TFIID complex, along with Pol2 and the general transcription factors [13]. Given the 247 importance of CHD1 in regulating PIC assembly and ultimately gene expression, we have 248 conducted further analyses to study the relationship between CHD1 binding and the length of $\mathrm{H} 3 \mathrm{~K} 27 \mathrm{ac}$ and H3K4me3 domains. Our analyses showed that there is a positive correlation between the length of CHD1 and the lengths of both H3K27ac and H3K4me3 domains (Fig. 4), implying that elongated CHD1 binding takes place at regions where longer H3K4me3 and longer H3K27ac deposition is observed. Broad H3K4me3 domains have been associated with increased and robust expression of genes, similarly genes marked by longer H3K27ac domains (i.e., super/stretch enhancers) have been associated with increased expression. Our findings show that longer H3K4me3 and H3K27ac domains have longer deposition of CHD1 binding; which might imply a more robust assembly of mediator complex and PIC enabled by the elongated binding of CHD1 protein. This robust assembly of PIC at these loci might explain their robust and increased expression patterns.

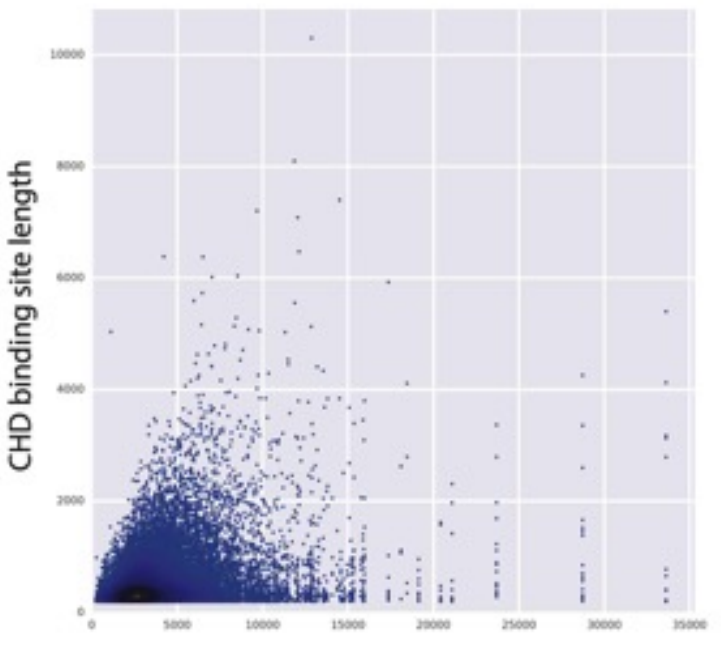

H3K4me3 domain length

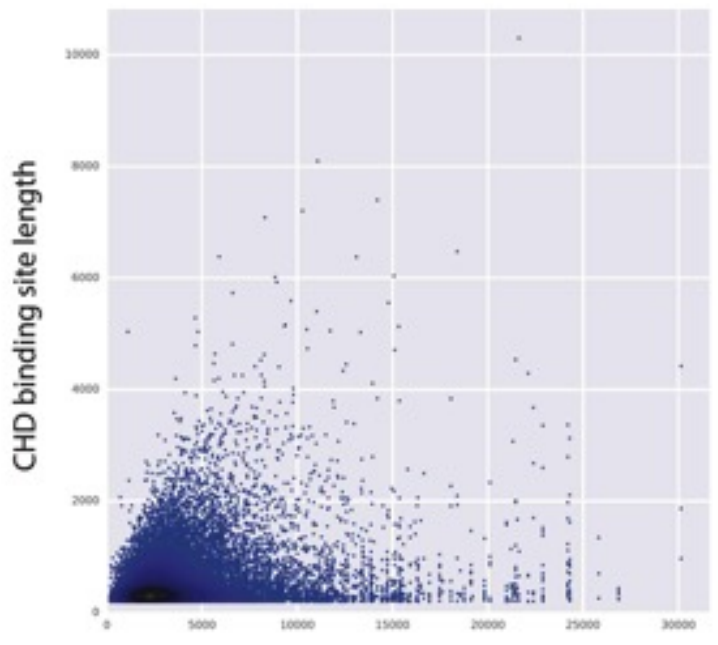

H3K27ac domain length

Figure 4: CHD1 binding length and H3K4me3 (and H3K27ac) domain lengths are correlated. $X$ axes represents the length of $\mathrm{H} 3 \mathrm{~K} 4 \mathrm{me} 3$ domains (left panel) and the length of $\mathrm{H} 3 \mathrm{~K} 27 \mathrm{ac}$ domains (right panel). $\mathrm{Y}$ axes represents the length of CHD1 bound loci.

\section{Broader H3K4me3 and H3K27ac are bounded by CTCF mediated interactions}

CTCF, is a TF that is important for the insulator activity. Insulators are boundary elements that restrict the regulatory interactions between enhancers and promoters within a boundary element. An intriguing hypothesis based on the high predictive value of CTCF for the length of H3K4me3 and H3K27ac domains is that the longer H3K27ac and H3K4me3 domains tend to be enriched at the boundary of chromatin domains marked by the CTCF binding. To test this, we analyzed CTCF mediated chromatin interactions obtained by the Chromatin Interaction Analysis by Paired-End Tag Sequencing (ChIA-PET) technology in GM12878 cell line [14]. We split H3K4me3 and H3K27ac domains into five bins based on their domain length and for each of these bins, we studied the localization of CTCF mediated chromatin interactions with respect to the center of the domain overlapping the CTCF fragment. Our analyses revealed an interesting pattern, where we observed that with increasing length of H3K27ac and H3K4me3 domains, 
275 CTCF interactions tend to cluster at the beginning or at the end of the domains (Figure 5), which 276 implies that longer H3K4me3 and H3K27ac domains tend to be at the boundary of CTCF defined 277 boundary domains.

278

284

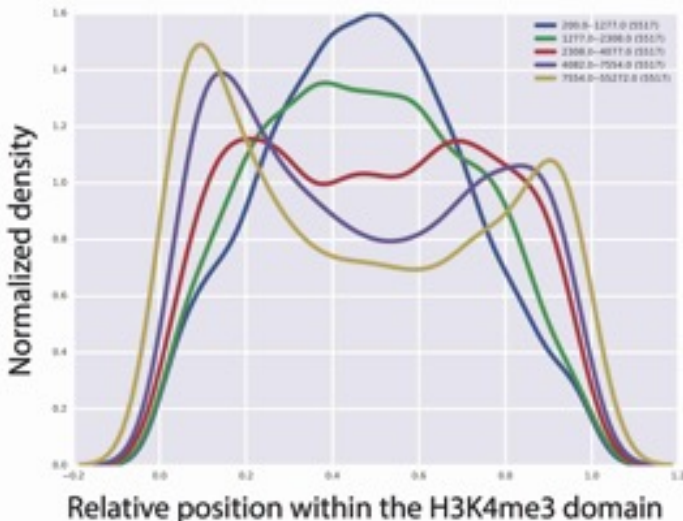

Figure 5: Localization of CTCF interactions over H3K4me3 (left panel) and H3K27ac (right panel) domains. X axis represents the relative position on the domains. $Y$ axis represents the CTCF interaction coverage. Yellow represents the longest domains, blue represents the shortest domains.

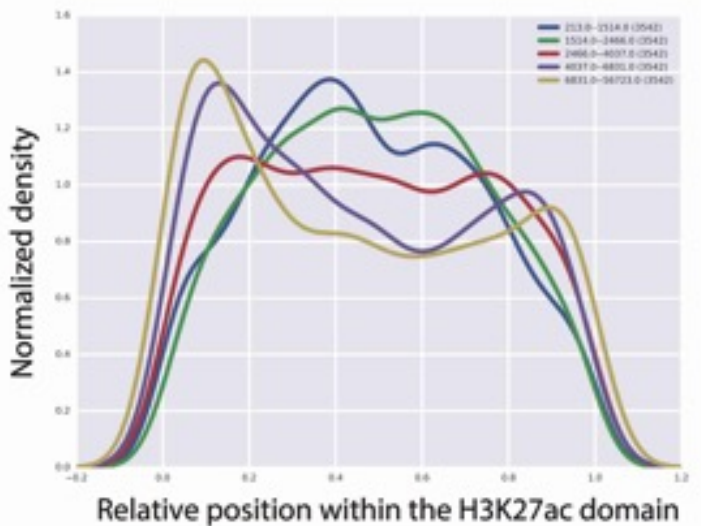

Relative position within the H3K27ac domain

\section{Discussion}

Recent epigenomic studies, including ours, have shown that the length of certain epigenetic mark deposition domains - H3K4me3 for promoters and H3K27ac for enhancers - might have functional implications and can be predictive in identifying promoters and enhancers that are critical for the underlying cell's identity and function. However, we still do not know the mechanisms that establish and maintain the boundaries of these domains, therefore it is still intriguing to investigate whether these domain lengths correlated with other genomic and epigenomic datasets using regression models, which might be important players in setting and maintaining these domains. Our analyses showed that in fact these domain lengths could be predicted with high accuracy using publically available Transcription Factor (TF) binding and histone modification ChIP-seq datasets in seven ENCODE cell lines as well as in human pancreatic islet samples. This high accuracy of our regression models show that the length of these domains correlates well with the genomic and epigenomic states of these sites within a cell and not a byproduct of assays or analyses. We conducted further analyses to understand in which ways these important predictors might be related to broad H3K4me3 and H3K27ac domains. Our analyses revealed that the nature of the relationship is based on the genomic feature in consideration. Our analyses with CHD1 binding revealed that elongated CHD1 binding is associated with longer $\mathrm{H} 3 \mathrm{~K} 27 \mathrm{ac}$ and $\mathrm{H} 3 \mathrm{~K} 4 \mathrm{me} 3$ domains, which might contribute to the robust and increased expression of genes associated with these domains via a robust assembly of PIC. On the other hand, for CTCF domains, we noticed that broader H3K4me3 and H3K27ac domains tend to locate towards the edges of CTCF defined boundary elements. Although our computational analyses cannot infer causality in the establishment of CTCF domains and broader deposition of epigenetic marks, we still observe that the deposition of domain length is not independent of the regulatory boundaries defined by CTCF binding. 


\section{Conclusions}

313 Our analyses revealed that there are frequently occurring predictors for $\mathrm{H} 3 \mathrm{~K} 4 \mathrm{me} 3$ and $\mathrm{H} 3 \mathrm{~K} 27 \mathrm{ac}$ 314 domain lengths. Among the predictors we identified, CHD1-binding is important for the 315 prediction of both H3K27ac and H3K4me3 domain lengths. CHD1 is essential for the assembly of 316 preinitiation complexes (PIC) and ultimately for regulating gene expression levels. We have conducted further analyses to show that broader H3K4me3 and H3K27ac domains have elongated CHD1 binding, which might play a role in their robust and increased expression patterns In the future, it would be interesting to study the effect of CHD1 knockdown or precise targeting on the expression levels of genes marked by broad H3K4me3 and H3K27ac domains. We also showed that broader H3K4me3 and H3K27ac domains localize towards the end of the regulatory boundary elements defined by CTCF binding by studying chromatin interactions datasets generated by ChIA-PET technology. In summary, our computational analyses revealed interesting features of epigenetic mark length for two important histone modification marks (H3K4me3 and H3K27ac) and showed in which ways these signals correlate with TF binding patterns and chromatin interactions. 


\section{References}

335

336

337

338

339

340

341

342

343

344

345

346

347

348

349

350

351

352

353

354

355

356

357

358

359

360

361

362

363

364

365

366

367

368

369

370

371

372

373

374

375

376

377

1. Hnisz, D., B.J. Abraham, T.I. Lee, A. Lau, V. Saint-Andre, A.A. Sigova, H.A. Hoke, and R.A. Young, Super-enhancers in the control of cell identity and disease. Cell, 2013. 155(4): p. 934-47.PMCID: 3841062

2. Chapuy, B., M.R. McKeown, C.Y. Lin, S. Monti, M.G. Roemer, J. Qi, P.B. Rahl, H.H. Sun, K.T. Yeda, J.G. Doench, E. Reichert, A.L. Kung, S.J. Rodig, R.A. Young, M.A. Shipp, and J.E. Bradner, Discovery and characterization of super-enhancer-associated dependencies in diffuse large B cell lymphoma. Cancer Cell, 2013. 24(6): p. 77790.PMCID: 4018722

3. Parker, S.C., M.L. Stitzel, D.L. Taylor, J.M. Orozco, M.R. Erdos, J.A. Akiyama, K.L. van Bueren, P.S. Chines, N. Narisu, N.C.S. Program, B.L. Black, A. Visel, L.A. Pennacchio, F.S. Collins, A. National Institutes of Health Intramural Sequencing Center Comparative Sequencing Program, and N.C.S.P. Authors, Chromatin stretch enhancer states drive cell-specific gene regulation and harbor human disease risk variants. Proc Natl Acad Sci U S A, 2013. 110(44): p. 17921-6.PMCID: 3816444

4. Jeong, M., D. Sun, M. Luo, Y. Huang, G.A. Challen, Rodriguez B., Zhang X., Chavez L., Wang H., Hannah R., Kim S.B., Yang L., Ko M., Chen R., Göttgens B., Lee J.S., Gunaratne P., Godley L.A., Darlington G.J., Rao A., Li W., Goodell M.A., Large conserved domains of low DNA methylation maintained by Dnmt3a. Nat Genet, 2014. 46(1): p. 1723.PMCID: 3920905

5. Benayoun, B.A., E.A. Pollina, D. Ucar, S. Mahmoudi, K. Karra, E.D. Wong, K. Devarajan, A.C. Daugherty, A.B. Kundaje, and E. Mancini, H3K4me3 breadth is linked to cell identity and transcriptional consistency. Cell, 2014. 158(3): p. 673-688

6. Chen, K., Z. Chen, D. Wu, L. Zhang, X. Lin, J. Su, B. Rodriguez, Y. Xi, Z. Xia, and X. Chen, Broad H3K4me3 is associated with increased transcription elongation and enhancer activity at tumor-suppressor genes. Nature genetics, 2015

7. Ernst, J. and M. Kellis, Large-scale imputation of epigenomic datasets for systematic annotation of diverse human tissues. Nature biotechnology, 2015. 33(4): p. 364-376

8. Hoffman, M.M., J. Ernst, S.P. Wilder, A. Kundaje, R.S. Harris, M. Libbrecht, B. Giardine, P.M. Ellenbogen, J.A. Bilmes, E. Birney, R.C. Hardison, I. Dunham, M. Kellis, and W.S. Noble, Integrative annotation of chromatin elements from ENCODE data. Nucleic Acids Res, 2013. 41(2): p. 827-41.PMCID: 3553955

9. Langmead, B., C. Trapnell, M. Pop, and S.L. Salzberg, Ultrafast and memory-efficient alignment of short DNA sequences to the human genome. Genome Biol, 2009. 10(3): p. R25.PMCID: 2690996

10. Pedregosa, F., G. Varoquaux, A. Gramfort, V. Michel, B. Thirion, O. Grisel, M. Blondel, P. Prettenhofer, R. Weiss, V. Dubourg, J. Vanderplas, A. Passos, D. Cournapeau, M. Brucher, M. Perrot, and E. Duchesnay, Scikit-learn: Machine Learning in Python. Journal of Machine Learning Research, 2011. 12: p. 2825-2830

11. Bernstein, B.E., A. Meissner, and E.S. Lander, The mammalian epigenome. Cell, 2007. 128(4): p. 669-81

12. Gaspar-Maia, A., A. Alajem, F. Polesso, R. Sridharan, M.J. Mason, A. Heidersbach, J. Ramalho-Santos, M.T. McManus, K. Plath, and E. Meshorer, Chd1 regulates open 
378

379

380

381

382

383

384

385

386

387

388

389

390 chromatin and pluripotency of embryonic stem cells. Nature, 2009. 460(7257): p. 863-868

13. Lin, J.J., L.W. Lehmann, G. Bonora, R. Sridharan, A.A. Vashisht, N. Tran, K. Plath, J.A. Wohlschlegel, and M. Carey, Mediator coordinates PIC assembly with recruitment of CHD1. Genes \& development, 2011. 25(20): p. 2198-2209

14. Tang, Z., Luo O.J., Li X., Zheng M., Zhu J.J., Szalaj P., Trzaskoma P., Magalska A., Wlodarczyk J., Ruszczycki B., Michalski P., Piecuch E., Wang P., Wang D., Tian S.Z., Penrad-Mobayed M., Sachs L.M., Ruan X., Wei C.L., Liu E.T., Wilczynski G.M., Plewczynski D., Li G., Ruan Y., CTCF-Mediated Human 3D Genome Architecture Reveals Chromatin Topology for Transcription. Cell, 2015. 163(7): p. 1611-27 